médecine/sciences $1991 ; 7: 22-9$

\title{
Le cancer médullaire de la thyroïde : acquis et interrogations
}

Le cancer médullaire de la thyroïde est une affection rare et grave dont au moins un quart des cas apparaît familial, lié à un locus situé sur le chromosome 10. Ces formes familiales ont une transmission dominante et, comme les formes sporadiques, peuvent soit se manifester par des cancers isolés de la thyroïde, soit s'intégrer à une néoplasie endocrinienne multiple qui pourra associer à la tumeur thyroïdienne des phéochromocytomes, des névromes sous-muqueux et sous-cutanés et un syndrome malformatif. Sur le plan anatomopathologique, le cancer médullaire de la thyroïde est caractérisé par une hyperplasie des cellules $\mathrm{C}$, bilatérale dans les formes familiales, l'existence d'un stroma amyloïde et la présence de calcitonine dans les cellules tumorales. L'hypersécrétion de cette hormone, spontanément ou après stimulation, constitue l'élément biologique le plus caractéristique, permettant souvent de faire le diagnostic de formes infracliniques.

\section{Claude Calmettes}

\section{ADRESSE}

C. Calmettes : docteur en médecine, AIHP, directeur de recherche. Inserm U. 113, faculté

de médecine Saint-Antoine, 27, rue Chaligny, 75571 Paris Cedex 12.

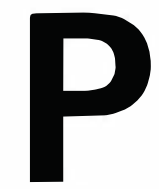

armi les cancers anaplasiques de la thyroïde, Hazard individualisait, en 1959 [1], le cancer médullaire de la thyroïde (CMT) sur l'existence d'un stroma amyloïde au sein de tumeurs d'évolution relativement lente. Depuis lors, ce cancer s'est affirmé remarquable tant par sa rareté et son polymorphisme clinique - il paraît isolé ou comme élément d'une polyendocrinopathie de type II - que par son caractère exceptionnel de cancer endocrinien à transmission héréditaire ou apparemment sporadique, doté d'un marqueur biologique, la calcitonine (CT).

Les données présentées dans ce texte tiennent largement compte des réflexions et des résultats du travail collaboratif mené au sein du Groupe d'Etude des Tumeurs à Calcitonine (GETC) depuis 1983.

\section{Historique}

Il n'est pas dans notre propos de refaire l'historique exhaustif de cette affection. Trois phases marquent son histoire et sa compréhension.

La première a été descriptive et il importe de rappeler que Pagès [2], rarement cité, avait reconnu dès 1955 l'existence de cancers thyroïdiens à cellules claires. En 1961, Sipple [3] individualise l'association phéochromocytome-cancer thyroïdien, qui portera son nom. Parallèlement, la CT est découverte par Hirsch [4] en 1963. La connaissance du cancer médullaire de la thyroïde progresse 
dès lors très rapidement : Williams montre que le cancer médullaire est en cause dans le syndrome de Sipple [5], Schimcke et Hartmann [6] suspectent son caractère familial puis Williams [7] suggère que le cancer se développe aux dépens des cellules parafolliculaires de la thyroïde, que Pearse [8] nomme cellules C.

En 1968, la constatation par Milhaud
[9] de la sécrétion de CT par ce cancer allait permettre le diagnostic et le suivi de la maladie; elle ouvrait le champ, après l'authentification de sa forme familiale la même année par Steiner [10], à sa détection précoce grâce à la réponse pathologique spécifique de l'hormone à des sécrétagogues [11]. Les descriptions des formes de la maladie allaient se succé- der : syndrome de Gorlin [12], néoplasies endocriniennes multiples (NEM) de type II, a et b. Par ailleurs, l'étude immunochimique de la CT dans les pièces d'exérèse thyroïdienne faisait découvrir une hyperplasie des cellules $\mathrm{C}$ dans la forme héréditaire [13] et des formes histologiques atypiques jusqu'alors méconnues [14].

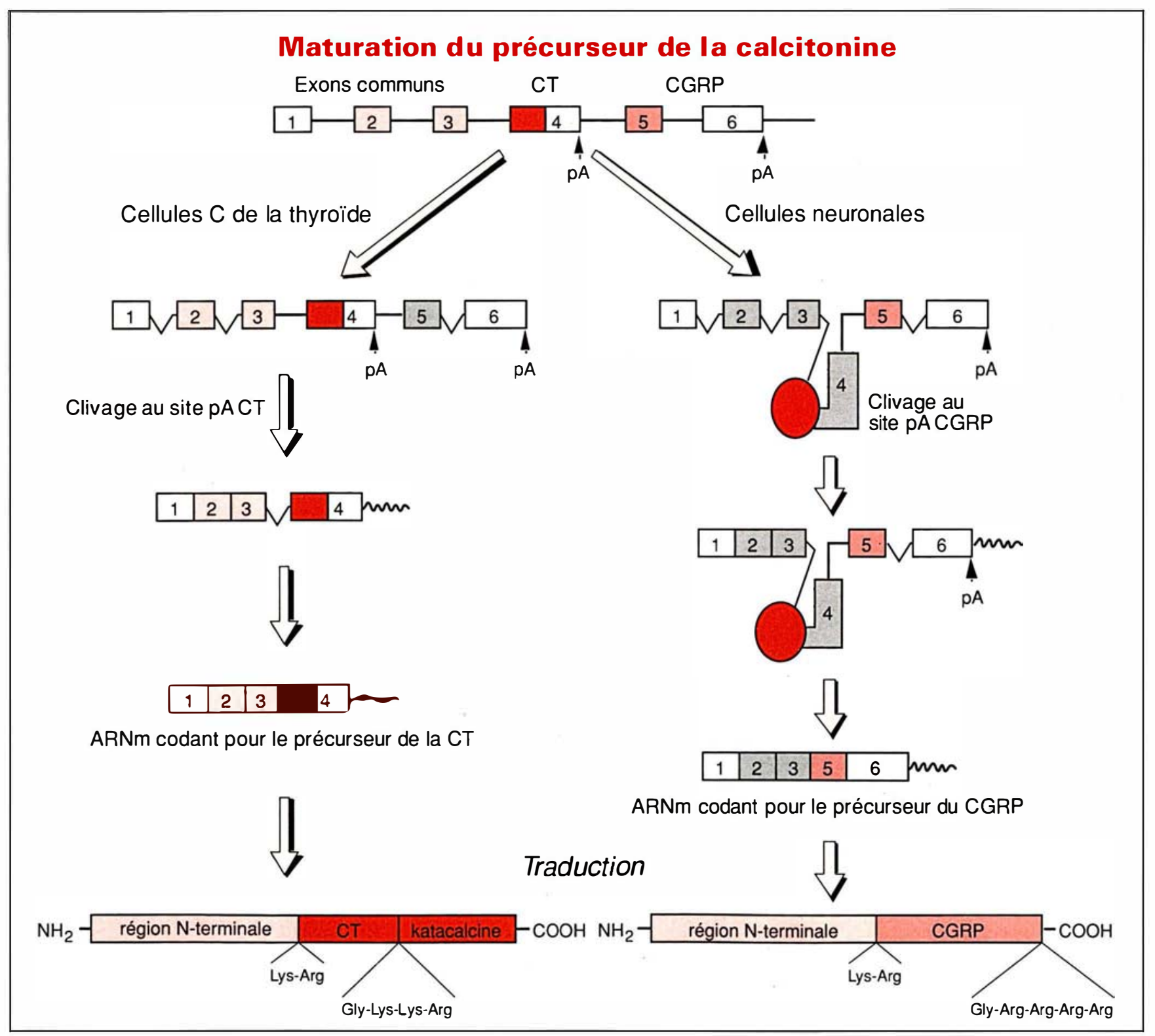

Figure 1. Modèle d'épissage avec intervention d'un facteur spécifique (ovale rouge) qui engage l'épissage dans la voie de formation de I'ARNm du CGRP. Les parties roses et rouges représentent les régions codantes, pA représente le site de polyadénylation (D'après [32] et [33]). 


\section{RÉFÉRENCES}

1. Hazard JB, Hawk WA, Crile Jr G Medullary (solid) carcinoma of the thyroid : a clinicopathologic entity. J Clin Endocrinol Metab 1959 ; 19: 152-61.

2. Pagès A. Essai sur le système des cellules claires de Feyrter. Thèse de doctorat en médecine, Montpellier, 1955.

3. Sipple JH. The association of pheochromocytoma with carcinoma of the thyroid gland. Am J Med 1961; 31 : 163-6.

4. Hirsch PF, Voelkel EF, Munson PL Thyrocalcitonin : hypocalcemic hypophos phatemic principle of the thyroid gland. Science $1964 ; 146$ : 412-3.

5. Williams ED. A review of 17 cases of carcinoma of the thyroid and pheochromocytoma. J Clin Path 1965 ; 288-92.

6. Schimke RN, Hartman WH. Familia amyloid-producing medullary thyroid carcinoma and pheochromocytoma : a distinct genetic entity. Am J Int Med 1965; 63 : 1027-39.

7. Williams ED. Histogenesis of medullary carcinoma of the thyroid. J Clin Path 1966 $19: 114-8$.

8. Pearse AGE. Common cytochemical properties of cell producing hormones, with particular reference to calcitonin and the thyroid C cells. Vet Rec $1966 ; 79$ : 587-90.

9. Milhaud G, Tubiana M, Parmentier C Coutris G. Epithélioma de la thyroïde sécrétant de la thyrocalcitonine. CR Acad Sci Paris $1968 ; 266$ : 608-10

10. Steiner AL, Goodman AD, Powers SR. Study of a kindred with pheochromocytoma, medullary thyroid carcinoma, hyperparathyroidism and Cushing's disease : multiple endocrine neoplasia. Medicine 1968; 47 . 371-409.

11. Hennessy JF, Gray TK, Cooper CW, Ontjes DA. Stimulation of thyrocalcitonin secretion by pentagastrin and calcium in two patients with medullary carcinoma of the thyroid. J Clin Endocrinol Metab 1973 ;
Une dernière phase est marquée par l'application au CMT des techniques de la génétique moléculaire, avec: la découverte du gène de la CT sur le chromosome 11 ; la mise en évidence de l'épissage alternatif (figure 1) de son transcrit primaire en un messager codant pour la CT, la katacalcine et le peptide $\mathrm{N}$-terminal, d'une part, un messager codant pour le calcitonin gene-related peptide (CGRP) [15], d'autre part, la localisation du (ou d'un) gène responsable de la transmission héréditaire de la maladie sur le chromosome 10 [16].

restant habituellement décelée par dosage de la calcémie et de la phosphorémie.

Les polyendocrinopathies de type IIb sont d'un extrême polymorphisme ; elles associent, en particulier et de façon variable, CMT bilatéral, phéochromocytomes, névromes diffus sous-cutanés et sous-muqueux et anomalies du développement ostéomusculaire qui deviendront de plus en plus manifestes au cours de la croissance. L'hyperplasie des plexus nerveux digestifs peut entraîner un pseudosyndrome de Hirschsprung**, révélateur de la maladie dès les premiers jours de la vie. Le CMT est particulièrement agressif dans ce cadre évolutif. La plupart des cas de NEM IIb sont apparemment sporadiques.

Dans une même famille peuvent exister des cas de cancer isolé et de polyendocrinopathies de types I et II. Deux composantes des CMT sont ainsi à mettre en exergue :

- l'existence des formes héréditaires qui exige formellement la recherche systématique d'autres cas dans la famille d'un malade ;

- la possibilité de l'association d'un phéochromocytome qui, méconnu, peut être à l'origine d'accidents dramatiques.

\section{- Forme familiale}

La détection d'un deuxième cas dans la famille est nécessaire et suffisante, du fait de la rareté de la maladie (1/25 000 sujets en Angleterre et Pays de Galles), pour en porter le diagnostic ; ses caractères - bilatéralité du cancer, hyperplasie des cellules $\mathrm{C}$, association à d'autres atteintes endocriniennes - ne sont pas formels puisqu'il existe des polyendocrinopathies apparemment sporadiques ayant les mêmes particularités. Il ne faut pas compter sur l'existence d'une symptomatologie familiale suspecte pour en faire le diagnostic (Tableau I) car la pénétrance clinique est incomplète. Il convient de pratiquer chez tous les apparentés au $1^{\text {er }}$ degré, quel que soit leur âge, un dosage de CT avec stimulation qui rarement le signe d'appel de la maladie ; le syndrome de Sipple est une affection essentiellement héréditaire qui touche la majorité des familles atteintes par la maladie. Les manifestations cliniques d'une hyperparathyroïdie associée sont rares, celle-ci

\footnotetext{
* Bouffées vasomotrices du visage.

** Maladie de Hirschsprung : mégacôlon congénital entraînant des syndromes occlusifs néonataux.
} 
Tableau I

CIRCONSTANCES DU DIAGNOSTIC DE LA FORME HÉRÉDITAIRE DU CANCER MÉDULLAIRE

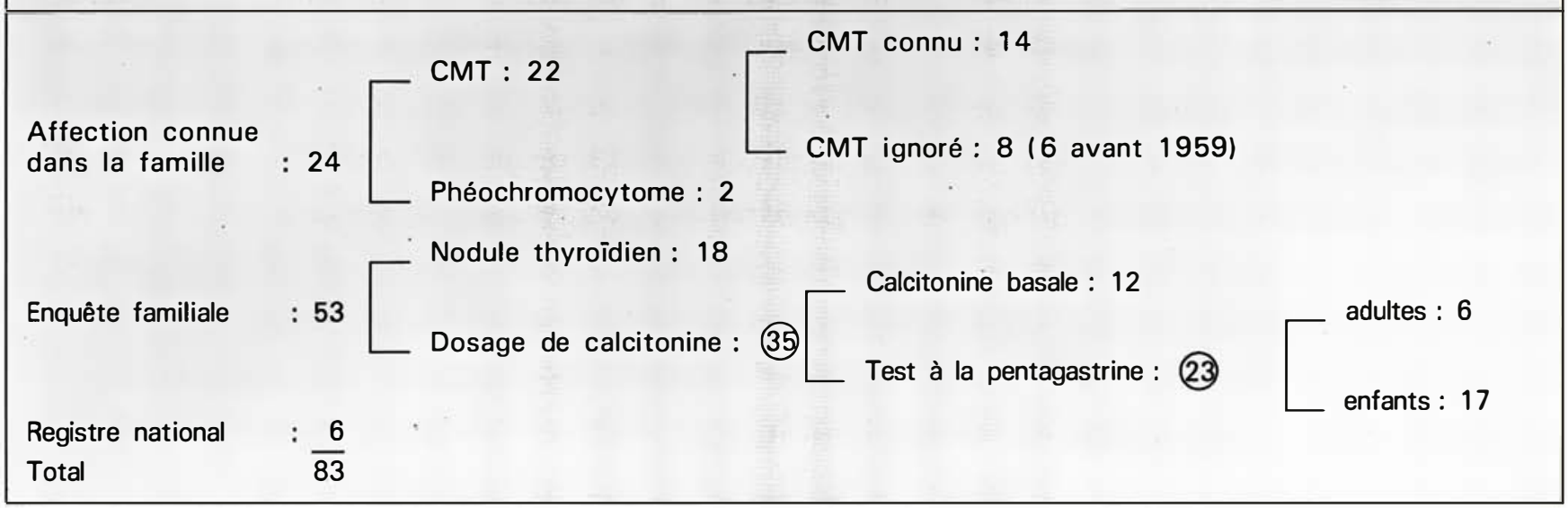

Dans 77 cas, un deuxième cas a été identifié dans la famille des malades, tumeur opérée (24 fois) ou formes ignorées et diagnostiquées à partir d'éléments indirects. Six fois, c'est l'apparentement de deux cas à partir du registre national qui a permis d'aboutir à un diagnostic de forme familiale. CMT : cancer médullaire de la thyroïde.

en permettra le diagnostic avant tout signe clinique. Le diagnostic de formes sporadique ne sera donc qu'un diagnostic d'exclusion et sa probabilité tiendra compte du nombre de sujets explorés, de leur répartition sur l'arbre généalogique, de leur âge (l'âge d'expression de la maladie est variable et $50 \%$ des sujets seulement ont révélé la maladie à 12 ans [17]) et des critères anatomopathologiques. C'est dire qu'il n'est pas toujours possible (famille restreinte, se refusant à l'enquête...) de faire la distinction entre formes familiale et sporadique.

\section{- Biologie}

La calcitonine est un marqueur fiable et sensible du CMT; c'est un polypeptide de 32 acides aminés, d'un poids moléculaire de l'ordre de 3 500. Elle circule sous forme de monomère et de dimère, ainsi que de fragments et de haut poids moléculaires, précurseurs, agrégats ou hormone liée [18].

Le gène de la CT est localisé sur le bras court du chromosome 11 [19], proche des gènes de la parathormone, de la tyrosine hydroxylase et de la somédine C. Sa structure est bien connue; l'analyse de la séquence du gène a mis en évidence la présence d'une séquence codante pour une autre protéine, [15] (le CGRP). Il est composé de 6 exons ; les 3 premiers sont communs aux deux précurseurs, l'exon 4 est spécifique de la CT et les exons 5 et 6 du CGRP (figure 1). La maturation post-transcriptionnelle des ARN est soumise à un phénomène d'épissage alternatif variable selon les tissus, avec codage préférentiel pour la CT dans la thyroïde et pour le CGRP dans le cerveau; dans le CMT, la maturation de l'ADN aboutit au double codage de la CT et du CGRP. Un $2^{e}$ gène de la $\mathrm{CT}$, de même structure, situé sur le bras long du chromosome 11 [20], ne conduit pas à la production de CT mais conduit à la production du ß-CGRP (CGRP II). L'existence d'un $3^{e}$ gène humain, codant pour une CT proche de celle des vertébrés inférieurs, a été postulée par Lasmoles [21] qui a mis en évidence dans le CMT des ARNm hybridables avec une sonde spécifique du messager de la CT aviaire.

La valeur diagnostique du dosage de la CT n'est plus à démontrer. Son taux est toujours pathologique dans les CMT à manifestation clinique ; sa réponse anormale à des sécrétagogues spécifiques, dont le calcium et la pentagastrine, permet d'identifier son origine thyroïdienne et d'éliminer une sécrétion hormonale tumorale ectopique [21] liée, par exemple, à un cancer bronchique. Son dosage permet donc de faire le diagnostic biologique d'un CMT thyroïdien ou métastatique.

Si le volume tumoral est minime, le taux basal de l'hormone peut être encore normal ; sa stimulation est alors indispensable pour détecter précocement, sur l'existence du pic spécifique ainsi déclenché, une récidive tumorale post-opératoire et, surtout, en exploration familiale, un microcancer ou une hyperplasie des cellules $\mathrm{C}$ prétumorale qui sont justiciables d'une thyroïdectomie totale. Le dosage des peptides flanquant la CT n'est pas utilisé en pratique courante : le peptide C-terminal ou katacalcine, produit de façon équimolaire avec la CT, n'apporte pas d'information complémentaire et la valeur diagnostique du peptide $\mathrm{N}$-terminal est encore à déterminer.

Le taux circulant de CGRP n'est élevé que si celui de la CT est pathologique, il ne répond pas aux sécrétagogues de la CT : il n'a donc pas d'intérêt diagnostique et ne permet pas de distinguer les formes sporadiques et familiales de la maladic.

Le polymorphisme de la CT entraîne des discordances entre les résultats des différents dosages. Le dosage biologique ne reconnaît que l'hormone biologiquement active, entière et non oxydée; les dosages radioimmunologiques font appel à des anticorps polyclonaux et détectent les formes hormonales correspondant aux 


\section{RÉFÉRENCES}

12. Gorlin A, Sedano HO, Vickers RA, Cervenka J. Multiple mucosal neuromas, pheochromocytoma and medullary carcinoma of the thyroid - a syndrome. Cancer $1968 ; 2: 293-9$.

13. Wolfe HJ, Melvin KEW, ServiSkinner SJ, et al. C-cell hyperplasia preceeding medullary thyroid carcinoma. $N$ Engl J Med 1973 ; 289 : 437-41.

14. Milhaud G, Calmettes C, Gilbert Dreyfus, Moukhtar MS. An unusual trabecular thyroid cancer producing calcitonin. Experentia (Basel) $1970 ; 26: 1381-3$

15. Amara SG, Jonas V, Rosenfeld MG, Ong ES, Evans RM. Alternative RNA processing in calcitonin gene expression generates mRNAs encoding different polypeptide products. Nature 1982 ; 298 : 240-4.

16. Mathew CGP, Chin KS, Easton DF, et al. A linked genetic marker for multiple endocrine neoplasia type $2 \mathrm{~A}$ on chromosome 10. Nature 1987 ; 328 : 527-8.

17. Gagel RF, Jackson CE, Block MA, et al. Age related probability of development of hereditary medullary thyroid carcinoma. J Pedial 1982 ; 101: 941-6.

18. Calmettes C, Moukhtar MS. Calcitonin in health and disease. In : Endocrinology of calcium metabolism. Parsons JA, ed. New York: Raven Press, 1982 : 211-33.

19. Hoppener JWM, Steenbergh PH, Zandberg J, et al. Localization of the polymorphic human calcitonin gene on chromosome 11. Hum Genet 1984; 66: 309-12.

20. Steenberg PH, Hoppener JWM, Zandberg J, Lips CJM, Jansz HS. A second human calcitonin/CGRP gene. FEBS Letl 1985 ; $183: 403-7$.

21. Lasmoles F, Jullienne A, Day F, Minvielle S, Milhaud G, Moukhtar MS. Elucidation of the nucleotide sequence of chicken calcitonin mRNA. Direct evidence for the expression of a lower vertebrate calcitonin-like gene in man and rat. Embo $J 1985 ; 4: 2603-7$ différents sites de liaison de ces anticorps ; le dosage radio-immunométrique à l'aide d'anticorps monoclonaux permet la détection de l'hormone mature grâce à la sélection de deux anticorps monoclonaux [22]. Il n'est pas possible d'établir de facteur de conversion entre les différents dosages car les formes de l'hormone circulante [23] varient d'un malade à l'autre, voire d'un moment à l'autre de l'évolution et selon l'éloignement du point de prélèvement par rapport à la source de production... Il est donc nécessaire d'interpréter les résultats d'un dosage en fonction de ces données, en soulignant que le dosage de l'hormone mature est le plus sensible et détecte un hypercalcitonisme basal antérieurement aux autres dosages.

L'élévation du taux d'antigène carcino-embryonnaire (ACE) ne peut que corroborer le diagnostic de CMT ; elle est inconstante, fait défaut au début de l'évolution tumorale mais peut précéder l'augmentation du taux de CT en cas de récidive tumorale : l'ACE constitue donc un marqueur essentiellement évolutif mais c'est aussi un marqueur sélectif du CMT dans le cadre des tumeurs endocrines.

L'ACTH peut être présente dans les cellules $\mathrm{C}$ tumorales et hyperplasiques et sa sécrétion responsable du syndrome de Cushing, rare - 18 cas recensés en France en vingt ans mais parfois révélateur du cancer thyroïdien.

De multiples hormones, peptides et enzymes [24] peuvent s'associer à la CT, telles l'histamine, la sérotonine, la $\gamma$-énolase, la DOPA carboxylase, la somatostatine, la bombésine, sans traduction clinique habituelle.

\section{- Imagerie}

Elle a pour but de faire un bilan d'extension, le diagnostic de l'affection étant biologique; les tumeurs accompagnées d'un taux basal normal ou supranormal de CT restent en dessous des possibilités de l'imagerie - qui n'est donc pas indiquée dans ces cas.

L'imagerie des surrénales permet le diagnostic et la localisation non seulement des phéochromocytomes mais aussi d'une hyperplasie surrénalienne, détectable par la mono- iodobenzylguanidine radiomarquée, qui peut en être le premier stade évolutif.

\section{- Thérapeutique}

Elle est essentiellement chirurgicale, thyroïdectomie totale bilatérale avec curage ganglionnaire de principe [25], a u minimum cervical bilatéral avec conservation des veines jugulaires et médiastinales supérieures, plus étendu en fonction des données de l'imagerie préopératoire et des résultats de l'examen ganglionnaire per-opératoire.

Les indications d'une chirurgie itérative et de la radiothérapie de lésions résiduelles localisées comme d'une thérapeutique adjuvante sont à discuter en fonction des données cliniques et biologiques ; leur succès reste limité.

\section{- Anatomopathologie}

Si la présence d'un stroma amyloïde a permis d'identifier le CMT, elle est inconstante; la présence seule de $\mathrm{CT}$, in situ, affirmera l'existence du cancer, même dans les formes typiques qui peuvent être simulées par des tumeurs à thyroglobuline, mais surtout dans les formes atypiques, trabéculovésiculaires parfois mixtes, anaplasiques, à cellules géantes ou mucosécrétantes.

L'anatomopathologie permet dans une large mesure la discrimination entre formes familiale et sporadique, les premières étant caractérisées par la bilatéralité des lésions et une hyperplasie annexe des cellules $\mathrm{C}$; la restriction vient du fait qu'elles ont ces éléments en commun avec les polyendocrinopathies de type II apparemment sporadiques. Il importe donc d'utiliser un protocole d'examen très strict.

\section{- Pronostic}

Le pronostic du CMT reste sombre dans les tumeurs de détection clinique, du fait de la précocité des métastases ganglionnaires dont l'exérèse cervicale et médiastinale supérieure n'entraîne de rémission complète que dans $90 \%$ des cas environ, mais il est tempéré par une évolution souvent lente qui peut dépasser vingt ans malgré la présence de localisations métastatiques multiples. Dans une même famille, le pronostic peut 
être différent d'un malade à l'autre et d'une fratrie à l'autre [26]. En revanche, la précocité du diagnostic dans les formes familiales transforme l'évolution de la maladie, comme en témoignent des études récentes [25, 27] (Tableau II) et nos dernières observations; aucun des 8 malades opérés avec un taux normal de CT mature, sur la foi d'un pic hormonal après pentagastrine, n'avait de métastase ; il s'agissait de 6 cancers bilatéraux, de 1 à $4 \mathrm{~mm}$ de diamètre, 5 familiaux et le dernier détecté lors de l'exploration d'un phéochromocytome, et de 2 cas d'hyperplasie des cellules $\mathrm{C}$, dont un cas de NEM IIb.

\section{- Études généalogiques}

Cette approche est mise en œuvre depuis un an à l'échelon national grâce à A. Chaventré (Ined, institut national d'études démographiques); elle a été axée sur la recherche de l' ancêtre chez qui est apparue la première mutation ("tête de mutation") pour les familles identifiées, d'une parenté entre sujets de même patronyme et d'une appartenance à des familles connues de cas apparemment sporadiques mais dont les caractères cliniques et/ou anatomopathologiques pouvaient faire suspecter une hérédité tumorale. Les conséquences en sont largement bénéfiques pour les malades :

- la réunion de branches de familles dispersées permet de limiter "en parapluie " l'exploration familiale aux descendants de l'ancêtre commun; - la réunion de familles souffrant de formes apparemment différentes de la maladie, CMT apparemment isolé et NEM IIa, voire NEM IIb (voir enca$d r e ́, \quad p .29)$, oriente vers l'existence d'un même gène à l'origine de formes cliniquement différentes et permet de suspecter et de rechercher de façon exhaustive une atteinte glandulaire plurifocale [25] dans des familles qui en semblaient jusque-là exemptes ;

- le diagnostic de la forme familiale a pu être porté par réunion de cas apparemment sporadiques ou par rattachement familial, avec reprise bénéfique de l'enquête familiale ;

- un cas de transmetteur "biologiquement et cliniquement sain ", âgé de plus de 50 ans, a été identifié; $m / s n^{\circ} 1$, vol. 7, janvier 91
Tableau ॥

CANCER MÉDULLAIRE DE LA THYROÏDE : IMPACT DU DIAGNOSTIC PRÉCOCE SUR L'EXTENSION GANGLIONNAIRE

\begin{tabular}{|l|c|c|c|}
\hline \multirow{2}{*}{ Métastases } & \multicolumn{3}{|c|}{ Type de cancer } \\
\cline { 2 - 4 } & Sporadique & \multicolumn{2}{|c|}{ Familial } \\
\cline { 2 - 4 } & & clinique & occulte \\
\cline { 2 - 4 } & 168 & 41 & 50 \\
\hline Macroscopiques & $52 \%$ & $41 \%$ & $2 \%$ \\
Microscopiques & $16 \%$ & $32 \%$ & $6 \%$ \\
\hline Total & $68 \%$ & $73 \%$ & $8 \%$ \\
\hline
\end{tabular}

D'après Marmouzet et al. [25].

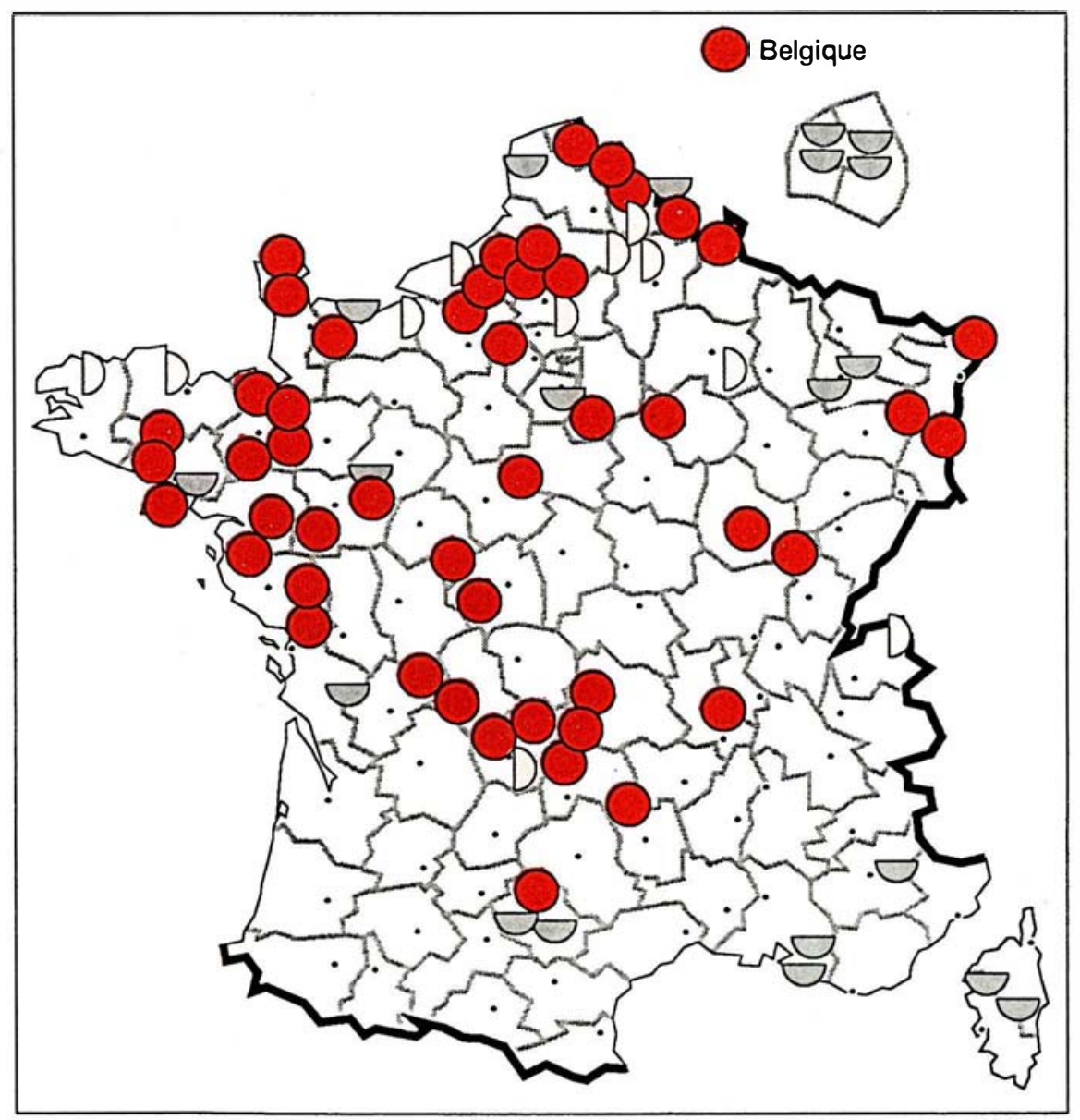

Figure 2. Localisation préliminaire des familles francaises atteintes de cancer médullaire de la thyroïde. 


\section{RÉFÉRENCES}

22. Motte P, Vauzelle P, Gardet P, et al. Construction and clinical validation of sensitive and specific assay for serum mature calcitonin using monoclonal anti-peptide antibodies. Clin Chim Acta 1988; 174 : 35-54.

23. Moukhtar MS, Jullienne A, Taboulet J, Calmettes C, Raulais D, Milhaud MS. Hétérogénéité de la calcitonine immunoréactive dans le plasma de sujets avec cancer médullaire. Pathol Biol 1975; 23 : 809-14.

24. Guliana JM, Modigliani E. Les marqueurs tumoraux du cancer médullaire de la thyroïde. Leurs aspects fondamentaux et endocriniens. Ann Endocrinol 1988; 49 : 34-50.

25. Marmousez T, Mellière D, Proye C, Calmettes C. Actualisation de la prise en charge chirurgicale des cancers médullaires de la thyroïde (tumeurs thyroïdiennes à calcitonine). Lyon Chir 1990; 86 : 104-7.

26. Houdent Ch, Avronsart B, Dubuisson $\mathrm{M}$, et al. Cancer médullaire familial de la thyroïde. Apport de la génćalogie et de la génétique à l'étude de deux familles. Presse Méd 1990 ; 19 : 549-52.

27. Guliana JM, Modigliani E, Guillausseau PJ, et al. Détection et pronostic du cancer médullaire de la thyroïde. Influence d'une collaboration multidisciplinaire. Presse Méd $1989 ; 18$ : 521-4.

28. Calmettes C, Ponder BAJ. Medullary carcinoma of the thyroid : proposal for collaboration in collection of clinical and epidemiological data within Europe. Copenhagen : EORTC Thyroid Study Group, 1986. (Abstr.)

29. Feingold N, Calmettes C. French medullary study group (GETC) : a first analysis of familial screening in apparently sporadic cases of MTC. Reims (France) EORTC Thyroid Study Group, 1987. (Abstr.)

30. Franc B. Le cancer médullaire de la thyroïde : acquisitions récentes. Sem Hop Paris 1990 ; 66 : 111-7.

31. Cote MY. De la recherche à la qualité des soins. médecine/sciences $1990 ; 6$ : 198-9.

32. Leff SE, Evans RM, Rosenfeld MG. Splice commitment dictates neuron-specific alternative RNA processing in calcitonin/CGRP gene expression. Cell $1987 ; 48$ : 517-24.

33. Emerson RB, Hedjran F, Yeakley JM, Guise JW, Rosenfeld MG. Alternative production of calcitonin and CGRP mRNA is regulated at the calcitonin-specific splice
- une carte du CMT en France (figure 2) ainsi que des aires de localisation de la maladie ont été établies.

\section{- Épidémiologie : le CMT en France}

L'enquête épidémiologique a pris en compte les différents items relatifs à une tumeur, de type endocrine, sporadique ou familiale, isolée ou partie intégrante d'une NEM, et possédant un marqueur biologique ; elle a été pratiquée à partir d'un protocole européen [28] établi avec la collaboration de N. Feingold (Inserm U.155) et diffusé d'une manière aussi exhaustive que possible. L'un de ses premiers objectifs est l'identification des familles informatives. Les résultats pour la période 1968-1988 sont en cours d'interprétation; l'analyse des données n'a pas encore permis de parvenir à des conclusions concernant l'incidence et la prévalence de la maladie en France et seules des valeurs globales sur le nombre de cas seront retenues pour ce laps de temps ; 1315 cas ont été recensés, 992 apparemment sporadiques et 323 familiaux, soit 1 cas sur 4, groupés en 85 familles dont 11 étrangères, ainsi réparties : 44 souffrant d'un CMT apparemment isolé, 36 de NEM IIa et 5 de NEM IIb. Il est remarquable de voir comment, depuis lors, ce tableau s'est modifié, et ce grâce à l'exploration familiale et surrénalienne de plus en plus systématique, à la sensibilité du dosage de la calcitonine mature et aux études généalogiques. Le nombre des familles s'est accru notablement - 100 au début de 1990, 11 étrangères - non seulement par détection de nouveaux cas mais aussi par glissement de cas sporadiques, essentiellement NEM, vers des cas familiaux, tandis que la proportion des différentes formes se modifiait en donnant une prédominance aux NEM IIa (53) sur les CMT apparemment isolés (41) pour un nombre de NEM IIb toujours faible (6), tandis que le nombre total des cas s'élevait à 1593 . Il est vraisemblable que le nombre des NEM IIa apparemment sporadiques et des formes familiales isolées devrait continuer à décroître. Jusqu'à présent, aucun cas familial unilatéral n'a été diagnostiqué [29] ; aucun cas de phéochromocytome n'a précédé la manifestation biologique du CMT qui peut toutefois être encore à un stade infraclinique.

La partie génétique moléculaire de l'enquête a été confiée à G. Lenoir, elle ne sera pas traitée ici. Signalons seulement que les résultats obtenus ont permis de considérer comme positifs, dans certaines familles, des résultats "limites" de tests à la pentagastrine.

\section{Des questions restent posées dans tous les domaines}

En biologie, il n'existe pas de marqueur tumoral, stricto sensu, du CMT, puisque la calcitonine est un marqueur sécrétoire de la cellule $\mathrm{C}$; la signification de l'hétérogénéité des formes circulantes de la calcitonine, comme de la coexistence des formes du messager codant pour la CT et le CGRP, n'est pas éclaircie.

La sensibilité du dosage de la CT mature permet de détecter une discrète stimulation (pic normal obtenu inférieur à $30 \mathrm{pg} / \mathrm{ml}$ ) de l'hormone par la pentagastrine chez certains sujets normaux et chez des sujets affectés d'une hyperplasie des cellules $\mathrm{C}$, sans discrimination possible. La valeur diagnostique du dosage des précurseurs de la CT, du peptide Cterminal, du peptide $\mathrm{N}$-terminal, des CGRP I et II est à éclaircir. La signification de la sécrétion des différents peptides et hormones comme leurs relations n'est pas élucidée. Quant au dosage de la chromogranine dans la détection des phéochromocytomes, il est à l'étude.

L'étude de l'expression des messagers de la CT en vue d'établir sa valeur pronostique et diagnostique est également en cours. Il n'est pas impossible qu'une modification de l'épissage alternatif des ARNm de la CT puisse aboutir à une production exagérée de CGRP et on ne peut exclure l'existence de formes de CMT dans lesquelles la dédifférenciation cellulaire aboutirait à sa sécrétion préférentielle.

En anatomopathologie, les études systématiques menées, en particulier en France, sur les cellules $\mathrm{C}$ conduisent à discuter non seulement les critères d'hyperplasie des cellules $\mathrm{C}$ et de leur bénignité mais aussi leur 
NEUROENDOCRINOPATHIES MULTIPLES DE TYPE I (ORIGINE ENDODERMIQUE) OU DE TYPE II (ORIGINE NEUROECTODERMIQUE)

NEM TYPE 1 : Syndrome de Wermer

Héréditaire à transmission autosomique dominante (chromosome 11) ou sporadique, avec participation de 2 ( $1 / 3$ des cas), 3 ( $1 / 3$ des cas) ou 4 ( $1 / 4$ des cas) atteintes endocrines.

- Adénome parathyroïdien ou hyperplasie, atteinte plurifocale, dans $95 \%$ des cas

Hyperparathyroïdie avec hypercalcémie, hypophosphorémie, lithiase rénale, atteinte osseuse, taux de PTH élevé.

- Prolifération plurifocale du pancréas endocrine, bénigne ou maligne, dans 30 à $60 \%$ selon les séries. - des cellules A1 (gastrinomes le plus souvent malins, $50 \%$ environ) avec hypersécrétion de gastrine responsable d'un syndrome de Zollinger Ellison associant diarrhée et ulcères gastriques et duodénaux multiples ;

- des cellules bêta (insulinomes, $30 \%$ environ, parfois associés aux gastrinomes) avec hypersécrétion d'insuline et accidents hypoglycémiques ; - des cellules D1 (vipomes malins, rares) avec hypersécrétion de vasointestinal peptide (VIP), diarrhée, hypokaliémie ;

- des cellules A2 exceptionnellement, avec hypersécrétion de glucagon, des cellules D2 à PP...

- Adénome hypophysaire, dans 15 à $90 \%$ des cas selon les séries, avec prolifération

- des cellules somatotropes $(25 \%)$, responsables d'une acromégalie ;

- des cellules à prolactine $(60 \%)$, avec galactorrhée, aménorrhée ;

- des cellules corticotropes entraînant parfois un syndrome de Cushing, le plus souvent non sécrétantes

- des cellules thyréotropes avec hyperthyroïdie ; - des cellules à ACTH $(3 \%)$

- non fonctionnels.

- Tumeurs carcinoïdes, lipomes..., rarement (5\% des cas)

NEM DE TYPE IIa : syndrome de Sipple

Héréditaire à transmission autosomique dominante (chromosome 10), plus rarement apparemment sporadique, associant 2 ou 3 des affections suivantes

- Cancer médullaire de la thyroïde, bilatéral, parfois sensible à, diarrhée... avec $\mathrm{n}$ marqueur biologique, incluant la calcitonine.

- Adénome parathyroïdien, unique ou multiple (voir plus haut), paucisymptomatique.

- Phéochromocytome, unique ou multiple, bénin (95\% des cas) ou malin, malaises, flushs, hypertension artérielle, parfois hypotension orthostatique... mais souvent latent.

NEM DE TYPE IIb, ou III : syndrome de Gorlin

Apparemment sporadique plus souvent que familial à transmission autosomique dominante,

- Cancer médulaire de la thyroïde, bilatéral, très agressif

- Nevromatose digestive avec nevromes labiaux, gingivaux et/ou hyperplasie des plexus nerveux digestifs, pseudosyndrome de Hirschsprung souvent néonatal, avec occlusion, caractéristiques du syndrome.

- Hyperplasie des nerfs cornéens et urétéraux ; et sécheresse lacrymale

- Syndrome dysmorphique, avec aspect marfanoïde, maigreur, morphologie, longiligne, scoliose...

- Anomalies cutanées, tumeurs, taches café au lait, angiomes

- Phéochromocytome, environ une fois sur deux - Hyperparathyroïdie, exceptionnellement. signification et leur existence dans diverses conditions physiologiques et pathologiques, leurs relations avec les cellules vésiculaires [30]. La signification du stroma amyloïde est elle aussi à élucider.

L'épidémiologie génétique devra établir la prévalence et l'incidence de la maladie mais elle doit être critique car la détection des cas princeps comme des cas familiaux n'est exhaustive que depuis peu de temps. Elle doit s'intéresser aux facteurs d'expressivité (âge, clinique, agressivité...) de la maladie, les étudier en particulier au sein des familles ; restent posés les problèmes d'existence éventuelle de transmetteurs "biologiquement et cliniquement sains" comme de néomutations, gamétique(s) ou somatique(s), transmissibles ou à l'origine de polyendocrinopathies sporadiques.

Le gène (ou les) gène(s) responsable(s) CMT, maladie qui semble plus proche du modèle polypose colique que du rétinoblastome, reste(nt) à localiser précisément et la nature des mutations reste à identifier. Sont aussi à élucider les mécanismes de l'hyperplasie cellulaire prétumorale, de la tumorigenèse et de la raison pour laquelle l'atteinte médullosurrénale reste habituellement bénigne.

Le champ à explorer reste donc très vaste, sans même aborder deux points particuliers :

- la biologie de la cellule $\mathrm{C}$, décrite surtout par immunocytochimie et à partir de culture de cellules tumorales ;

- l'étude du modèle animal du CMT que constitue le rat Wag Rij qui développe un cancer spontané et permet ainsi de procéder à une étude biologique et anatomopathologique de la tumeur spontanée, de tumeurs greffées et de cultures cellulaires tumorales, ainsi qu'à des essais thérapeutiques. Le cancer médullaire de la thyroïde est une maladie rare; les progrès réalisés dans sa connaissance témoignent de la valeur d'une "intégration disciplinaire " - associant des spécialistes de toutes disciplines comme des praticiens généralistes - et du rôle de la recherche : "recherche fondamentale, clinique ou épidémiologique, recherche évaluative, organisationnelle et opérationnelle ou recherche sociale appliquée" [31].
Parallèlement, la formation des publics et la vulgarisation des données entraînent une détection plus précoce des formes familiales et une amélioration du pronostic de l'affection

\section{Summary}

Medullary thyroid cancer

Medullary thyroid carcinoma (MTC) is a particularly interesting model of the gene expression in cancer. Progress in the knowledge and early cure of this rare disease benefits from a close collaboration between practitioners and scientists.

The description of the different forms, MTC only and multiple endocrine neoplasia (MEN II), either sporadic or familial, with the discovery of calcitonin (CT) as a specific marker of the tumor, has been improved in the last decade by different events :

- The localization of the CT gene on chromosome 11, the alternative splicing of its messengers and the secretion of CGRP I, CGRP II, salmon-type CT and other hormones and peptides by MTC.

- The discovery of an abnormality responsible for the familial disease linked to chromosome 10 . - The improvement of the hormone assay, by the use of monoclonal antibodies, resulting in a more precocious MTC diagnosis. A nation-wide French study has allowed an extensive diagnosis of the familial form (up to 100). 'The concomitant use of genealogical studies resulted not only in their recognition by linkage of patients but also in joining of family branches, with diagnosis of MEN syndrome, limiting the screening to the descendants of the common ancestor... An epidemiological work is in progress for the period 1968-1988.

\section{TIRÉS A PART}

C. Calmettes. 